\title{
Myocardial perfusion imaging in very elderly patients with suspected coronary artery disease: Never too late!
}

\author{
Pasquale Perrone-Filardi, MD, PhD, ${ }^{\mathrm{a}}$ Alberto Cuocolo, $\mathrm{MD},{ }^{\mathrm{b}}$ \\ and Santo Dellegrottaglie, $M D, P^{c, d}$
}

\section{See related article, pp. 244-255}

Increasing longevity and declining fertility are the factors mainly responsible for the rapid growth of geriatric population in Western countries during the past few decades. Subjects born during the post-World War II baby boom are now starting to reach geriatric age, expanding the demographic group of the elderly. In the United States, the proportion of the population in the senior age group ( $\geq 65$ years) is expected to peak at just over $20 \%$ in 2030 , while in the time interval from the years 2000-2030, the absolute number of the very old (>85 years of age) will double from 9.3 to 19.5 million. ${ }^{1,2}$ This will result in a sizeable increase in coronary artery disease (CAD) prevalence, since aging is a significant cardiovascular risk factor. ${ }^{3,4} \mathrm{CAD}$ is the most common cause of death in the elderly, but its diagnosis and management may be particularly challenging in aged patients. In fact, due to age-related changes in the cardiovascular system, reduced multi-system organ reserve as well cardiovascular and non-cardiovascular comorbidities, clinical presentation can be misleading in aged patients.

Despite evidence of higher benefit of pharmacologic and revascularization therapies in older compared to younger patients, community practice patterns continue to indicate a limited use in elderly patients. ${ }^{5}$ At

From the Department of Clinical Medicine, Cardiovascular and Immunological Sciences, ${ }^{\text {a }}$ Section of Cardiac Imaging, Department of Biomorphological and Functional Sciences, ${ }^{\mathrm{b}}$ Federico II University, Naples, Italy; Division of Cardiology, ${ }^{\mathrm{c}}$ Ospedale MedicoChirurgico Accreditato Villa dei Fiori, Naples, Italy; and Z. and M.A. Wiener Cardiovascular Institute and M.-J. and H.R. Kravis Center for Cardiovascular Health, ${ }^{\mathrm{d}}$ Mount Sinai Medical Center, New York, NY.

Reprint requests: Pasquale Perrone-Filardi, MD, PhD, Department of Clinical Medicine, Cardiovascular and Immunological Sciences, Federico II University, Via Pansini 5, 80131 Naples, Italy; fpperron@unina.it.

J Nucl Cardiol 2012;19:224-6.

$1071-3581 / \$ 34.00$

Copyright ( 2011 American Society of Nuclear Cardiology.

doi:10.1007/s12350-011-9499-0 least, in part, this is due to the concern that elderly are generally more vulnerable to the adverse effects of drugs used in CAD, as well as the procedural complications of revascularization, and to the fact that only few randomized clinical trials verified the efficacy of treatment strategies of CAD in elderly patients. ${ }^{6}$

Yet, there is no doubt that revascularization improves prognosis in high-risk old patients. The Trial of Invasive Versus Medical Therapy in Elderly Patients (TIME) study enrolled patients over 75 years of age with stable angina and randomly assigned them to a conservative strategy (optimal medical therapy) or an invasive strategy (coronary angiography and revascularization when appropriate). ${ }^{7}$ At short-term follow-up (6 months), the conservative strategy was associated with less favorable results on angina severity and quality of life, with an increased risk of major adverse cardiac events $(49 \%$ vs $19 \%, P<.001)$. In further analyses involving the TIME population, the invasive strategy remained associated with a significantly better prognosis at 1-year follow-up, but longer observations revealed a similar survival in the two groups. ${ }^{8,9}$

In a sub-group analysis of the Clinical Outcomes Utilizing and Aggressive Drug Evaluation (COURAGE) study focusing on patients aged $\geq 65$, the addition of percutaneous coronary intervention (PCI) to optimal medical therapy did not impact on clinical outcomes in the whole cohort of elderly patients during a median 4.6-year followup. ${ }^{10}$ The nuclear sub-study of COURAGE indicated that significant reduction in risk of death or myocardial infarction is observed in patients with $\geq 5 \%$ reduction in myocardial ischemia with no interaction of age. ${ }^{11}$

The diagnostic and prognostic values of single photon emission computed tomography (SPECT) myocardial perfusion imaging (MPI) have been clearly established during the last three decades, with the extent and severity of myocardial hypoperfusion shown to be independent predictors of prognosis in patients with known or suspected CAD. ${ }^{12-14}$ However, only a minority of the clinical studies conducted with SPECT MPI so far have been focused on elderly patients. ${ }^{15}$ Diagnostic accuracy seems to be preserved and, at least at the shortterm follow-up, normal or mildly abnormal SPECT MPI 
appears to identify elderly patients at low risk of major adverse cardiac events. Even less investigated is the role of stress myocardial SPECT MPI for the diagnostic and prognostic evaluations of CAD in very old patients. Isolated reports conducted in small groups of patients indicated that even in very old patients, a negative SPECT MPI study is associated with a relatively low risk of subsequent cardiac events. ${ }^{16}$

In this issue of the Journal, Nair et $\mathrm{al}^{17}$ describe the prognostic value of SPECT MPI in very old patients with suspected CAD. They reported a retrospective evaluation of 1,093 patients $\geq 80$ years of age out of a total population of 8,864 consecutive patients with suspected CAD aged 50 years and older. Patients with evidence of CAD were excluded. The study cohort was categorized into three age groups: middle-aged (5064 years of age), elderly (65-79 years of age), and very elderly ( $\geq 80$ years of age). In each age category, the results of SPECT MPI were reported using the summed stress score (SSS) and the summed difference score (SDS). After the SPECT MPI study, major adverse cardiac events (cardiac death and non-fatal myocardial infarction) were recorded during a mean follow-up of $1.9 \pm .9$ years. SPECT MPI results were made available to the referring physician and contributed to select management strategy in each patient.

In this study, several types of stress were used as indicated by the referring physician. ${ }^{17}$ This resulted in significant differences between age groups, with vasodilator stress testing more frequently used in the very elderly than in elderly and middle-aged patients (63\% vs $35 \%$ and $20 \%$, respectively; $P<.001)$. In contrast, exercise stress testing was less commonly applied in the elderly than in the other age category $(10 \%$ vs $34 \%$ and $56 \%$, respectively; $P<.001)$. The preference in the very elderly for non-exercise stress protocols has been already reported in other studies and appears to be a direct consequence of the reduced ability to exercise because of functional limitation or comorbidities. ${ }^{18}$ As a consequence, the applied type of stress may already select patient populations with different levels of risk (generally higher in the group undergoing pharmacological stress SPECT MPI), as demonstrated by the very low event rate $(.7 \%$ per year) observed in the study for patients who were able to exercise and who had a negative MPI. This outcome was more favorable than observed in the entire cohort of elderly patients with a negative MPI (1.8\% per year). ${ }^{17}$

As expected, a higher rate of cardiac events was observed in the very elderly as compared to other age categories. ${ }^{17}$ Using Cox regression in the very elderly patients, SSS was identified as a significant multivariable predictor of adverse cardiac events (HR 1.090, 95\% CI 1.047-1.135, $P<.001)$. In addition, Cox proportional- hazards modeling revealed that the inclusion of the variable SSS added to traditional clinical and stress-ECG data, increases the ability to predict adverse cardiac events in very elderly patients. These data are of extreme relevance since they fill the considerable gap existing in the evidence base supporting the value of SPECT MPI for prognostic stratification in elderly patients.

Hachamovitch et $\mathrm{al}^{19}$ published the principal study so far available investigating the prognostic value of SPECT MPI in the elderly. The study included 5,200 consecutive patients with 75 years of age or older undergoing rest thallium-201/stress technetium-99 m sestamibi dual-isotope SPECT MPI with exercise (42\%) or adenosine $(58 \%)$ stress and principally referred for CAD diagnosis. The fact that over $70 \%$ of the included patients had resting ECG abnormalities, leading to the impossibility of using stress-induced ECG changes for CAD diagnosis, emphasizes the value of SPECT MPI in this category of patients $(44 \%$ of the very elderly patients had resting ECG abnormalities in Nair et $\mathrm{al}^{17}$ ).

In the case of a normal SPECT MPI, Hachamovitch et al reported an unadjusted, annualized cardiac death rate of $1.0 \%$ per year in the 75-84-year group and of $3.3 \%$ per year in the $\geq 85$-year-old age group (about onethird lower than the age-adjusted rate in the general population) over a mean follow-up of $2.8 \pm 1.7$ years. In addition, an interaction between early treatment strategy and ischemia was detected in a sub-group of 684 patients with extended follow-up (6.2 \pm 2.9 years), suggesting that a high degree of ischemia was associated with increasing survival with early revascularization, whereas in the setting of little or no ischemia by SPECT MPI, medical therapy had improved outcomes. These findings are consistent with the results of the nuclear sub-study of the COURAGE Trial. ${ }^{11}$ In the study conducted by Nair et al ${ }^{17}$ in this issue of the Journal, very elderly patients showed a significant increase in early ( $\leq 60$ days after SPECT MPI results) cardiac catheterization and revascularization as a function of severity of myocardial ischemia on SPECT MPI. Interestingly, even though the very elderly showed a higher severity of myocardial ischemia, a clear propensity toward a conservative approach was observed when compared with younger patients. Compared with patients in the elderly and middle-age categories, the very elderly with severe ischemia (SDS > 7) were referred less frequently for early cardiac catheterization $(41 \%$ vs $57 \%$ and $59 \%$, respectively; $P<.001)$ as well as for early revascularization (26\% vs $45 \%$ and $40 \%$, respectively; $P<.001)$. Yet, it is also of concern that $>40 \%$ of younger patients with high-risk SPECT MPI did not undergo invasive evaluation.

Increasing use of drug-eluting stents, transradial access, and more accurate prevention of renal function 
impairment is expanding the use of PCI in very old patients. In a Scottish registry collecting 31,758 nonemergency PCIs, the proportion of elderly patients (age $\geq 75$ years) went from $8.7 \%$ in 2000 to $13.9 \%$ in 2007 although, when compared with younger patients, the elderly demonstrated a significantly higher risk of major cardiovascular events at an early follow-up. ${ }^{20}$ Similarly, a cohort study conducted in Denmark demonstrated that the annual proportion of patients $\geq 80$ years of age undergoing PCI for any reason almost doubled (from $5.4 \%$ to $10.2 \%$ ) during the period between 2000 and 2007. ${ }^{21}$ In the same time period, progress in the care of patients undergoing coronary artery bypass graft $(\mathrm{CABG})$ surgery led to improved outcomes in elderly patients, thereby reducing perioperative complications. ${ }^{22,23}$

Thus, effective risk stratification in the elderly is, and will increasingly be, much needed, and the study from Nair et al ${ }^{17}$ represents an important contribution to the delineation of the prognostic value of SPECT MPI in this setting of patients. The findings of this study should hopefully foster prospective multicenter trials to assess whether MPI-assisted management of elderly patients with suspected CAD will ultimately translate in a costeffective outcome benefit in this population.

\section{Conflict of interest}

Drs Perrone-Filardi, Cuocolo, and Dellegrottaglie have no disclosures to report.

\section{References}

1. Foot DK, Lewis RP, Pearson TA, Beller GA. Demographics and cardiology, 1950-2050. J Am Coll Cardiol 2000;35:1067-81.

2. Centers for Disease Control and Prevention. Trends in aging: United States and worldwide. MMWR 2003;52:101-4.

3. Wang TJ, Gona P, Larson MG, et al. Markers for prediction of first major cardiovascular events and death. N Engl J Med 2006;355: 2631-9.

4. Odden MC, Coxson PG, Moran A, Lightwood JM, Goldman L, Bibbins-Domingo $\mathrm{K}$. The impact of the aging population on coronary heart disease in the United States. Am J Med 2011;124:82733.

5. Foody JM, Roc MT, Chen AY, et al., for the CRUSADE Investigators. Lipid management in patients with unstable angina pectoris and non-ST-segment elevation acute myocardial infarction (from CRUSADE). Am J Cardiol 2005;95:483-5.

6. Capodanno D, Angiolillo DJ. Antithrombotic therapy in the elderly. J Am Coll Cardiol 2010;56:1683-92.

7. The TIME Investigator. Trial of Invasive versus Medical therapy in Elderly patients with chronic symptomatic coronary-artery disease (TIME): A randomized trial. Lancet 2001;358:951-7.

8. Pfisterer M. Trial of Invasive versus Medical therapy in Elderly patients Investigators. Long-term outcome in elderly patients with chronic angina managed invasively versus by optimized medical therapy: Four-year follow-up of the randomized Trial of Invasive versus Medical therapy in Elderly patients (TIME). Circulation 2004;110:1213-8.

9. Kaiser C, Kuster G, Erne P et al., for the TIME Investigators. Risks and benefits of optimized medical and revascularization therapy in elderly patients with angina - on-treatment analysis of the TIME trial. Eur Heart J 2004;25:1036-42.

10. Teo KK, Sedlis SP, Boden WE, et al. Optimal medical therapy with or without percutaneous coronary intervention in older patients with stable coronary disease: A pre-specified subset analysis of the COURAGE (Clinical Outcomes Utilizing Revascularization and Aggressive Drug Evaluation) trial. J Am Coll Cardiol 2009;54:1303-8.

11. Shaw LJ, Berman DS, Maron DJ, et al. COURAGE Investigators: Optimal medical therapy with or without percutaneous coronary intervention to reduce ischemic burden: results from the Clinical Outcomes Utilizing Revascularization and Aggressive Drug Evaluation (COURAGE) trial nuclear substudy. Circulation 2008;117:1283-91.

12. Brown KA, Boucher CA, Okada RD, et al. Prognostic value of exercise thallium-201 imaging in patients presenting for evaluation of chest pain. J Am Coll Cardiol 1983;1:994-1001.

13. Hachamovitch R, Berman DS, Shaw LJ, et al. Incremental prognostic value of myocardial perfusion single photon emission computed tomography for the prediction of cardiac death: Differential stratification for risk of cardiac death and myocardial infarction. Circulation 1998;97:535-43.

14. Gimelli A, Rossi G, Landi P, Marzullo P, Iervasi G, L'abbate A, Rovai D. Stress/rest myocardial perfusion abnormalities by gated SPECT: Still the best predictor of cardiac events in stable ischemic heart disease. J Nucl Med 2009;50:546-53.

15. Perrone-Filardi P, Costanzo P, Dellegrottaglie S, et al. Prognostic role of myocardial single photon emission computed tomography in the elderly. J Nucl Cardiol 2010;17:310-5.

16. Zafrir N, Mats I, Solodky A, Ben-Gal T, Sulkes J, Battler A. Prognostic value of stress myocardial perfusion imaging in octogenarian population. J Nucl Cardiol 2005;12:671-5.

17. Nair SU, Ahlberg AW, Mathur S, Katten DM, Polk DM, Heller GV. The clinical value of single photon emission computed tomography myocardial perfusion imaging in cardiac risk stratification of very elderly patients ( $\geq 80$ years) with suspected coronary artery disease. J Nucl Cardiol 2012; in press.

18. Hashimoto A, Palmer EL, Scott JA, et al. Complications of exercise and pharmacologic stress tests: Differences in younger and elderly patients. J Nucl Cardiol 1999;6:612-9.

19. Hachamovitch R, Kang X, Amanullah AM, et al. Prognostic implications of myocardial perfusion single-photon emission computed tomography in the elderly. Circulation 2009;120:2197-206.

20. Johnman C, Oldroyd KG, Mackay DF, et al. Percutaneous coronary intervention in the elderly: Changes in case-mix and periprocedural outcomes in 31,758 patients treated between 2000 and 2007. Circ Cardiovasc Interv 2010;3:341-5.

21. Antonsen L, Jensen LO, Thayssen P, et al. Comparison of outcomes of patients $\geq 80$ years of age having percutaneous coronary intervention according to presentation (stable vs unstable angina pectoris/non-ST-segment elevation myocardial infarction vs ST-segment elevation myocardial infarction). Am J Cardiol 2011;108:1395-400.

22. Alexander KP, Anstrom KJ, Muhlbaier LH, et al. Outcomes of cardiac surgery in patients $>$ or $=80$ years: results from the National Cardiovascular Network. J Am Coll Cardiol 2000;35:731-8.

23. Saxena A, Dinh DT, Yap CH, et al. Critical analysis of early and late outcomes after isolated coronary artery bypass surgery in elderly patients. Ann Thorac Surg 2011;92:1703-11. 